\title{
Identifying diamagnetic interactions in scattering and nonlinear optics
}

\author{
Kayn A. Forbes, David S. Bradshaw, and David L. Andrews* \\ School of Chemistry, University of East Anglia, Norwich NR4 7TJ, United Kingdom
}

(Received 21 July 2016; published 21 September 2016)

\begin{abstract}
In the generic formulation of optical interactions there is, beyond the familiar electric and magnetic multipolar forms of coupling, an additional diamagnetization term that rarely receives attention. In fact it can give rise to effects that should be observable in the general context of nonlinear optical spectroscopy, as well as scattering. A quantum electrodynamical analysis reveals features of special interest in two specific cases: two-photon absorption and Rayleigh scattering. Diamagnetic contributions are seen to be dispersion free with regards to the frequency of input radiation, and can represent unique interactions within optical absorption and emission processes. There is also a configuration in which diamagnetic couplings, which are quadratic in the magnetic field, can supersede those that are dependent linearly on the electric field strength, such as the electric dipole. In this connection the influence of retroreflected circularly polarized light, which leads to a local distance dependence in magnitude of the electromagnetic fields, produces conditions in which the diamagnetization response can become a prominent feature in two-photon absorption.
\end{abstract}

\section{INTRODUCTION}

In the field of electrodynamics, optical processes are often studied under the constraint of the electric-dipole approximation, wherein the wavelength of the incident or emergent light is assumed to be much larger than the dimensions of the irradiated particle. Under this supposition, the spatial variation of the vector potential is neglected. In consequence, higher-order couplings expressed in multipolar form, such as the light-matter interactions mediated by a magnetic dipole, can usually be disregarded. However, these higher-order terms are important for certain systems, such as chiral discrimination in molecules of low symmetry [1,2], light-harvesting complexes [3], nanomaterials [4-6], metamaterials [7,8] and numerous theoretical studies including optical trapping [9-14]. Such terms also assume greater significance for systems in which electric-dipole couplings are either very small or vanish altogether-when, for example, a relevant electronic transition is electric-dipole forbidden by symmetry. In fact, at a level of magnitude approximately two orders smaller than electric-dipole coupling, there are contributions to the quantum amplitude from both magnetic dipole and electric quadrupole terms, both of which are linear in the radiation field. Recent research on thin films has even shown that, at judiciously chosen wavelengths, higher-order nonlinear effects can be larger than linear [15]. However, as will be shown, another form of electromagnetic coupling can also become important under certain circumstances, as discussed in the following.

In addition to electric and magnetic multipolar couplings, one contribution that is very rarely discussed, or else assumed unimportant, is the diamagnetic interaction. As we shall see, this has many unique properties - including a quadratic dependence on the electromagnetic field, so that its importance grows with higher light intensities. Moreover, since each field interaction involves the creation or annihilation of a photon, it follows that this diamagnetic term is always present in any process that fundamentally involves more than one

\footnotetext{
*david.andrews@physics.org
}

photon event - for example, any form of light scattering or multiphoton absorption. Indeed, the diamagnetic interaction plays a part in every nonlinear optical interaction, by each and every particle of matter.

This paper begins by laying down the theoretical foundation for the diamagnetic form of interaction, starting with a Lagrangian representation of the electrodynamics and moving to the interaction Hamiltonian, thereby identifying the common origin of the diamagnetic interaction alongside the more commonly known electric dipole and other higher-order multipoles. The lowest-order diamagnetic interaction Hamiltonian is presented, and we then use it to study and develop the specific cases of two-photon absorption and Rayleigh scattering. By application of the Fermi rule, fully rotationally averaged rates are derived and presented for both of these processes, and it is shown how the rate expressions can be cast in terms of more familiar electric-dipole transition moments. We finish with a discussion highlighting the dispersive-free nature of the diamagnetic interactions, also showing how by exploiting retroreflection of circularly polarized beams it should be possible to make diamagnetic contributions dominant in any given multiphoton process.

\section{THEORETICAL FOUNDATION}

The treatment of particles and fields in a single electrodynamical system, in which energy can exchange between radiation and matter, can be achieved either through a Lagrangian or Hamiltonian formulation. The starting point for any quantum electrodynamical framework is usually the adoption of the minimal coupling Lagrangian or the multipolar Lagrangian, in either case followed by canonical transformation to the respective Hamiltonian form [16]. In its application to molecules, the advantage of the multipolar Hamiltonian over the minimal coupling formulation is the fact that, in the former, intermolecular Coulombic (instantaneous) interactions are eliminated so that these interactions are mediated solely by photons. Moreover, the minimal coupling method introduces unnecessary additional complexity when examining radiationmolecule and molecule-molecule interactions [17]. A strong 
case can be made that the multipole approach more directly delivers physical insights with regard to selection rules: As a result, this article focuses on the multipolar Hamiltonian formulation. With the aim of highlighting the origin of the diamagnetic interaction, a concise derivation from the corresponding Lagrangian is outlined below. More detailed derivations can be sourced elsewhere $[16,17]$.

The total multipolar Lagrangian can be partitioned into a sum of three terms: one for the molecules, $L_{\text {mol }}$ (labeled by $\xi$ ), another for the radiation, $L_{\mathrm{rad}}$, and the third for their interactions, $L_{\text {int }}$. The molecular components are written in terms of particles $\alpha$ with coordinates $\boldsymbol{q}_{\alpha}(\xi)$ and velocities $\dot{q}_{\alpha}(\xi)$, and the vector potential field $\boldsymbol{a}(\boldsymbol{r})$ with time derivative $\dot{\boldsymbol{a}}(\boldsymbol{r})$. In the absence of interaction between molecules and radiation, $L_{\mathrm{mol}}$ and $L_{\mathrm{rad}}$ are simply the particle Lagrangian and the free-field Lagrangian, whose respective dynamical variables bear no influence on one another. However, in a single dynamical system where the equations of motion of both matter and radiation are dependent on one another, the coupling appears as the interaction term $L_{\text {int }}$. The explicit form of the multipolar Lagrangian is givenby

$$
L_{\text {mult }}=L_{\mathrm{mol}}+L_{\mathrm{rad}}+L_{\mathrm{int}}
$$

in which

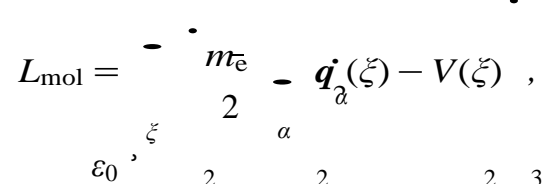

$$
\begin{aligned}
& \left.L_{\mathrm{rad}}=-\frac{\boldsymbol{a}}{2}(\boldsymbol{r})-c[\nabla \times \boldsymbol{a}(\boldsymbol{r})]\right\} d \boldsymbol{r}, \\
& L_{\text {int }}=[\nabla \times \boldsymbol{m}(\boldsymbol{r})] \cdot \boldsymbol{a}(\boldsymbol{r}) d^{3} \boldsymbol{r} \\
& -\quad \boldsymbol{p}^{\perp}(\boldsymbol{r}) \cdot \dot{\boldsymbol{a}}(\boldsymbol{r}) d^{3} \boldsymbol{r}-\underset{\xi<\xi^{\mathrm{r}}}{-} V_{\mathrm{inter}}\left(\xi, \xi^{r}\right) .
\end{aligned}
$$

Here $V(\xi)$ is the intramolecular Coulomb potential energy of molecule $\xi$, summing all the internal Coulombic interactions, and $V_{\text {inter }}\left(\xi, \xi^{r}\right)$ is the intermolecular energy between molecules $\xi$ and $\xi^{r}$. In Eq. (4), $\boldsymbol{m}(\boldsymbol{r})$ is the magnetization field that is related to the current density of a medium and $\boldsymbol{p}^{\perp}(\boldsymbol{r})$ is the transverse component of the electric polarization field [18], which also involves the charge distribution of a medium.

Changing from the multipolar Lagrangian to the multipolar Hamiltonian requires a canonical transformation of Eq. (1). This basically means that $\dot{\boldsymbol{q}}$ and $\dot{\boldsymbol{a}}$ are eliminated and replaced by the canonical momenta $\boldsymbol{p}$ and $\boldsymbol{M}$, such that

$$
H_{\mathrm{mult}}={ }_{\xi, \alpha} \boldsymbol{p}_{\alpha}(\xi) \cdot \dot{\boldsymbol{q}}_{\alpha}(\xi)+\boldsymbol{M}(\boldsymbol{r}) \cdot \dot{\boldsymbol{a}}(\boldsymbol{r}) d^{3} \boldsymbol{r}-L_{\mathrm{mult}}
$$

The momentum $\boldsymbol{p}_{\alpha}(\xi)$ is canonically conjugate to the position vector $\boldsymbol{q}_{\alpha}(\xi)$, as determined by the partial derivative,

$$
\boldsymbol{p}_{\alpha}(\xi)=\frac{\partial L_{\text {mult }}}{\partial \dot{q}_{\alpha}(\xi)}=m \boldsymbol{q}_{\alpha}(\xi)-\boldsymbol{n}_{\alpha}(\xi, \boldsymbol{r}) \times \boldsymbol{b}(\boldsymbol{r}) d^{3} \boldsymbol{r},
$$

where the vector field, $\boldsymbol{n}_{\alpha}(\xi, \boldsymbol{r})$, is defined as

$$
\begin{aligned}
\boldsymbol{n}_{\alpha}(\xi, \boldsymbol{r})= & -e\left[\boldsymbol{q}_{\alpha}(\xi)-\boldsymbol{R}_{\xi}\right] \\
& \times{ }_{0}^{, 1} \lambda \delta\left[\boldsymbol{r}-\boldsymbol{R}_{\xi}-\lambda\left(\boldsymbol{q}_{\alpha}(\xi)-\boldsymbol{R}_{\xi}\right)\right] d \lambda .
\end{aligned}
$$

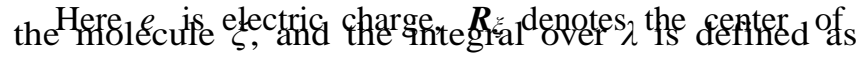
${ }_{0}^{1} \lambda^{n} d \lambda=(n+1)^{-1}$ (integration over this dimensionless parameter allows the multipole series to be expressed in a closed form), with $\boldsymbol{n}_{\alpha}(\xi, \boldsymbol{r})$ signifying a distribution for polarization that differs from $\boldsymbol{p}_{\alpha}(\xi, \boldsymbol{r})$ in its multipolar weightings. The momentum conjugate to the vector potential $\boldsymbol{a}(\boldsymbol{r})$ is

$$
\boldsymbol{M}(\boldsymbol{r})=\stackrel{\frac{\mathbb{Q}_{\text {mult }}}{\partial \dot{\boldsymbol{a}}}}{=}=\varepsilon_{0} \dot{\boldsymbol{a}}(\boldsymbol{r})-\boldsymbol{p}^{\perp}(\boldsymbol{r})=-\boldsymbol{d}^{\perp}(\boldsymbol{r}),
$$

where $\boldsymbol{d}^{\perp}(\boldsymbol{r})$ is the transverse electric displacement field. Now that the canonical momentum has been determined, we can substitute for $\dot{\boldsymbol{q}}$ and $\dot{\boldsymbol{a}}$ in the Legendre transformation, and by regrouping terms the following multipolar Hamiltonian is found:

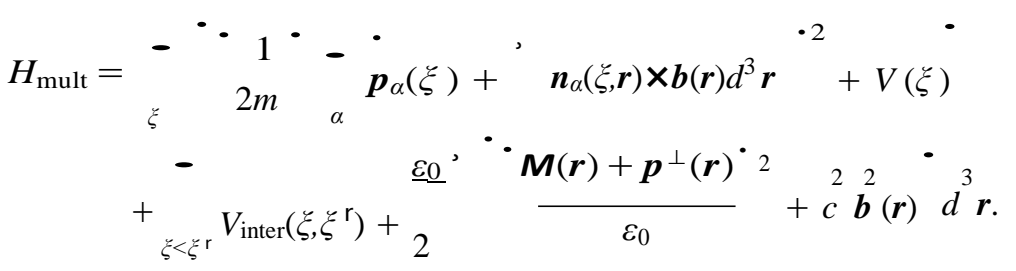

When using (6) and (8) to express the above equation in terms of $\dot{\boldsymbol{q}}_{\alpha}(\xi)$ and $\dot{\boldsymbol{a}}(\boldsymbol{r})$, it can be seen that this expression sums energy from the molecules and from the radiation field, where each of these terms is a sum of kinetic and potential energies. Thus the Hamiltonian takes the following form:

$$
H=(T+V)_{\mathrm{mol}}+(T+V)_{\mathrm{rad}} .
$$

It transpires that the intermolecular Coulomb interaction energy is exactly canceled by the intermolecular part of the transverse polarization. In consequence, it follows from Eq. (9) that the multipolar Hamiltonian becomes

$$
H_{\mathrm{mult}}=H_{\mathrm{mol}}+H_{\mathrm{rad}}+H_{\mathrm{int}}+\frac{1}{2 \varepsilon_{0}}{ }_{\xi}\left|\boldsymbol{p}^{\perp}(\xi, \boldsymbol{r})\right|^{2} d^{3} \boldsymbol{r},
$$

in which the final term is an intramolecular self-energy independent of the electromagnetic field. The absence of any contribution linking different molecules means that all intermolecular interactions are mediated by the transverse 
electromagnetic field - i.e., all such couplings are fully retarded. We now have

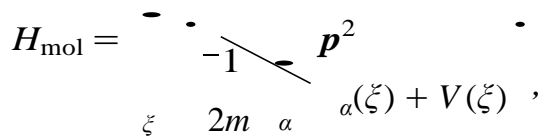

$$
\begin{aligned}
& H_{\mathrm{rad}}={ }_{2}^{1} \frac{\boldsymbol{M}^{2}(\boldsymbol{r})}{\varepsilon_{0}}+c^{2} \varepsilon_{0}[\nabla \times \boldsymbol{a}(\boldsymbol{r})]^{2} \quad \stackrel{3}{d} \boldsymbol{r}
\end{aligned}
$$

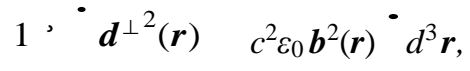

$$
\begin{aligned}
& =\varepsilon_{2} \quad \varepsilon_{0}+ \\
& H_{\text {int }}=\frac{1}{\delta_{0}} \quad \boldsymbol{p}^{\perp}(\boldsymbol{r}) \cdot \boldsymbol{M}(\boldsymbol{r}) d^{3} \boldsymbol{r}-\quad \boldsymbol{m}(\boldsymbol{r}) \cdot[\nabla \times a(\boldsymbol{r})] d^{3} \boldsymbol{r} \\
& +\frac{1}{2 m}^{\cdot}, \boldsymbol{n}(\boldsymbol{r}) \times[\nabla \times \boldsymbol{a}(\boldsymbol{r})] d^{3} \boldsymbol{r} \\
& =-{\frac{1}{\varepsilon_{0}}}^{\prime} \boldsymbol{p}(\boldsymbol{r}) \cdot \boldsymbol{d}^{\perp}(\boldsymbol{r}) d^{3} \boldsymbol{r}-{ }^{\prime} \boldsymbol{m}(\boldsymbol{r}) \cdot \boldsymbol{b}(\boldsymbol{r}) d^{3} \boldsymbol{r}
\end{aligned}
$$

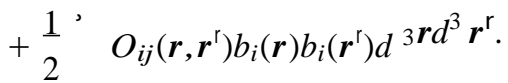

Here, the last term of Eq. (14) represents the diamagnetic interaction - the focus of interest in this paper-while the first two terms yield the electric and magnetic multipole expansions, respectively. It is interesting to observe that this term is quadratically dependent on an electromagnetic field, which represents an intrinsic nonlinearity in the interaction Hamiltonian. For deployment in a QED framework the status is clear; this term can contribute only to processes that entail two or more fundamental interactions. However, it should be borne in mind that such a distinction will be much less obvious in any semiclassical treatment, where the usual additivity of quantum amplitude contributions masks an assumed linear dependence on the fields.

To continue, the material tensor involved in the diamagnetic coupling is the local field $O_{i j}\left(\boldsymbol{r}, \boldsymbol{r}^{\mathrm{r}}\right)$, given explicitly by

$$
O_{i j}\left(\boldsymbol{r}, \boldsymbol{r}^{r}\right)={ }_{\xi, \boldsymbol{r}}^{-1} \varepsilon_{i k l} \varepsilon_{j m l} n_{k}(\xi, \boldsymbol{r}) n_{m}\left(\xi, \boldsymbol{r}^{\mathrm{r}}\right) .
$$

In most calculations, $H_{\text {mult }}$ is expanded in terms

multipole moments. While this is familiar for the electric and magnetic polarization fields, denoted by $\boldsymbol{p}(\boldsymbol{r})$ and $\boldsymbol{m}(\boldsymbol{r})$, respectively, the procedure can also be applied to $O_{i j}\left(\boldsymbol{r}, \boldsymbol{r}^{\mathrm{r}}\right)$. After carrying out the volume integral, the first few terms of the interaction portion of the multipolar Hamiltonian, $H_{\text {int }}^{\text {mult }}$, are identified as

$$
\begin{aligned}
H_{\mathrm{int}}^{\mathrm{mult}}= & -\cdot-\varepsilon_{0}^{-1} \boldsymbol{\mu}(\xi) \cdot \boldsymbol{d}^{\perp}\left(\boldsymbol{R}_{\xi}\right) \\
& \xi \\
& -{ }_{0} Q_{i j} Q_{i j}(\xi) \nabla_{j} d_{i}-\boldsymbol{m}(\xi) \cdot \boldsymbol{b}\left(\boldsymbol{R}_{\xi}\right) \\
& +\frac{e^{2}}{8 m} \underset{\xi, \alpha}{-}\left\{\left[\boldsymbol{q}_{\alpha}(\xi)-\boldsymbol{R}_{\xi}\right] \times \boldsymbol{b}\left(\boldsymbol{R}_{\xi}\right)\right\}^{2}+\cdots .
\end{aligned}
$$

Here, using the convention of implied summation over repeated Cartesian indices, the result is now written in explicit terms of the electric dipole, electric quadrupole, and magnetic dipole, and the final cross-product term represents the lowestorder diamagnetic coupling. Before proceeding further, it is worth noting some differences of the diamagnetization contribution from the more familiar electric quadrupole term - as it, too, is cast in terms of a second-rank coupling tensor. The latter, however, assumes a traceless form as a result of its engagement with the derivative of the electric field, whose components in the direction of the field itself vanish. (This is because, in the interaction term $Q_{i j} \nabla_{i} e_{j}$, the trace of $Q$ delivers $\nabla_{i} e_{\dot{b}}$ and while the gradient of the field lies in the direction of
propagation, the electric field is entirely transverse. In fact, the same comment can be made about the magnetic quadrupole term, since the magnetic field is intrinsically divergence free).

\section{DIAMAGNETIZATION IN OPTICAL INTERACTIONS}

In quantum electrodynamics, the mode expansion for the electromagnetic field operator $\boldsymbol{b}$ is given as

$$
\begin{aligned}
\boldsymbol{b}(\boldsymbol{r})= & i-\underline{\mathrm{k} k}^{\cdot 1 / 2} \\
& \boldsymbol{k}^{-\eta}{ }^{2 \varepsilon_{0} c V} \\
& \times\left\{\boldsymbol{b}{ }^{(\eta)}(\boldsymbol{k}) a^{(\eta)}(\boldsymbol{k}) e^{i \boldsymbol{k} \cdot \boldsymbol{r}}-\overline{\boldsymbol{b}}^{(\eta)}(\boldsymbol{k}) a^{+(\eta)}(\boldsymbol{k}) e^{-i \boldsymbol{k} \cdot \boldsymbol{r}}\right\},
\end{aligned}
$$

where $a^{(\eta)}(\boldsymbol{k})$ and $a^{\dagger(\eta)}(\boldsymbol{k})$ are the normalized annihilation and creation operators, respectively, for photons of the mode $(\boldsymbol{k}, \eta)$. The polarization vector $\boldsymbol{b}^{(\eta)}(\boldsymbol{k})$ is a unit vector in the direction of the magnetic field and $V$ is the quantization volume. Taking the final term of Eq. (16) with the mode expansion (17), we can rewrite the lowest-order diamagnetic contribution to the interaction Hamiltonian in terms of field components, using Levi-Civita symbols to express the vector cross products, as follows:

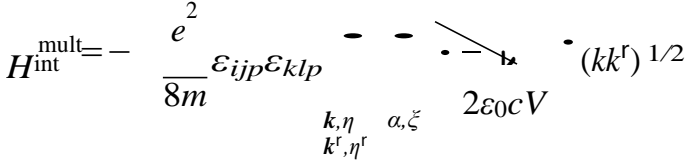

$$
\begin{aligned}
& \times\left\{\left[\boldsymbol{q}_{\alpha}(\xi)-\boldsymbol{R}_{\xi}\right]_{i}\left[\boldsymbol{q}_{\alpha}(\xi)-\boldsymbol{R}_{\xi}\right]_{k}\right\}
\end{aligned}
$$

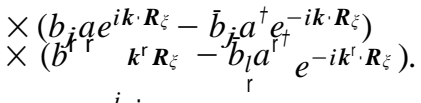

$$
\begin{aligned}
& a e^{i} \text {. }
\end{aligned}
$$

Here, the $\boldsymbol{k}$ and $\eta$ dependence of the photon annihilation and creation operators, along with the polarization vectors, are implicit. From the above result, it is immediately clear that the diamagnetic term can only participate in processes that involve two or more photons. The reason is that the photon creation and annihilation operators appear in quadratic combinations, emerging from the product of the last two bracketed terms in Eq. (18). Accordingly, if both of the photons relating to these operators are from the same radiation mode, the transition diamagnetization moment will depend on the square of the field strength, and the corresponding rate on the square of the radiation mode intensity. On the other hand, for a scattering process in which the two magnetic interactions relate to different radiation modes, this quadratic dependence on the input does not, of course, apply. We now consider these two cases in detail. 


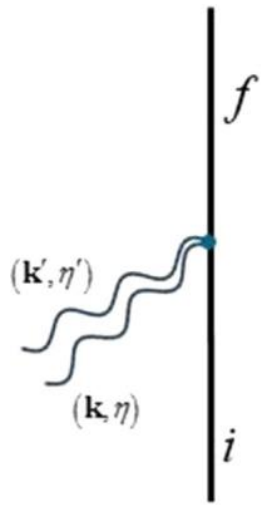

(a)

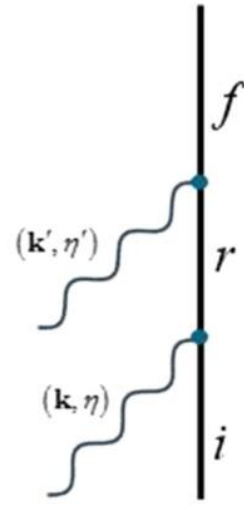

(b)
FIG. 1. (a) Feynman diagram for the diamagnetic two-photon absorption, where the molecule undergoes transition from an initial state $i$ to a final state $f$. (b) Conventional diagram for two-photon absorption, where $r$ is a virtual intermediate state.

\section{A. Application to two-photon absorption}

Two-photon absorption (TPA) is a well-studied process, widely used in nonlinear spectroscopy [19]. Single-beam TPA is a suitable process to exhibit how a scalar diamagnetic contribution can become both readily identifiable and dominant compared to other multipole contributions. We begin with standard perturbation theory, which tells us the leading contribution to the matrix element for the diamagnetic contribution to TPA is of first order in the interaction term. In certain respects this appears to be analogous to one-photon absorption because, in the diamagnetic contribution, both photons are annihilated at the same point on the world line in the Feynman graph, Fig. 1(a). In contrast, the leading contributions of the nondiamagnetic TPA terms, which originate from the other orders of multipole (including dipole) interactions, are second order in the interaction term, Fig. 1(b). Another key difference between the diamagnetic and nondiamagnetic contributions is

now evident: the lack of an intermediate state in diamagnetic interactions. It is an issue we shall return to later.

Let a beam of $n$ photons with mode $(\boldsymbol{k}, \eta)$ be incident upon a system of $N$ molecules each with an initial energy $E_{0}$. For such a case, the matrix element $M_{f i}$ for the diamagnetic contribution to TPA is given by

$$
M_{f i}=\left(f\left|H_{\mathrm{int}}\right| i\right),
$$

where $H_{\text {int }}$ is given by Eq. $(18)$ and $\left.\left.\left.\mid i\right)=\mid n(\boldsymbol{k}, \eta)\right) \mid E_{0}\right)$ and $\boldsymbol{f} \mid=\left(n-2(\boldsymbol{k}, \eta) \mid\left(E_{\boldsymbol{f}} \mid\right.\right.$. The radiation part of the matrix element is written as

$$
\begin{aligned}
(n & \left.-2(\mathbf{k}, \eta)\left|a^{(\eta)} a^{(\eta)}\right| n(\mathbf{k}, \eta)\right) \\
& =\sqrt{ } n\left(n-2(\mathbf{k}, \eta)\left|a^{(\eta)}\right| n-1(\mathbf{k}, \eta)\right) \\
& =n \overline{n-1}(n-2(\mathbf{k}, \eta) \mid n-2(\mathbf{k}, \eta)) \\
& =\{n(n-1)\}^{1 / 2} .
\end{aligned}
$$

On the usual assumption that the two-photon transition involves the promotion of only a single electron, and that its wave function is to a first approximation exactly separable from those of the other electrons, then it is possible to further develop the matrix element into a form that entails transition dipole moments. This simply involves use of the completeness relation to introduce a sum over states $\mid r)$, together with the defining operator relation $-e^{-}{ }_{\alpha}\left[\boldsymbol{q}_{\alpha}(\xi)-\boldsymbol{R}_{\xi}\right]_{i}=\mu_{i}(\xi)$. Hence we obtain

$$
M_{f i}=\varepsilon_{i j p} \varepsilon_{k l p} \frac{\mathrm{k} k}{16 m \varepsilon_{0} c V}\left\{n(n-1){ }_{r}^{1 / 2}{ }_{r}^{-} \mu_{i} \mu_{k} b_{j} b_{l},\right.
$$

where

$$
\begin{aligned}
\left(\mu_{i} \mu_{k}\right)^{f 0} & =\left(f\left|\mu_{i} \mu_{k}\right| 0\right)={ }_{r}^{-}\left(f\left|\mu_{i}\right| r\right)\left(r\left|\mu_{k}\right| 0\right) \\
& -\underset{r}{f r_{r} 0} \\
& =\underset{r}{\mu_{i} \mu_{k} .}
\end{aligned}
$$

To secure the rate, we now deploy the Fermi rule $T=$ $(2 \pi / k)\left|M_{f i}\right|^{2} N \rho_{f}$. In passing we observe that in applications to multiphoton absorption, the most appropriate density of states for application of this formula is the density of final states $\rho_{f}$ of the molecule, so that the result reduces to being dependent on the line shape of only the molecular states. Although in principle the density of states for any particular process will reflect a combination of uncertainties from both matter and radiation, it is the factor that contributes the highest overall number of states per unit frequency, momentum, or energy interval that will play the decisive role. For this reason-especially since multiphoton absorption is invariably studied with narrow-linewidth laser light — it is both expedient and appropriate to deploy a density of final material states for nonlinear absorption or other such excitation processes.

Continuing, the rate $T$ is thus given by

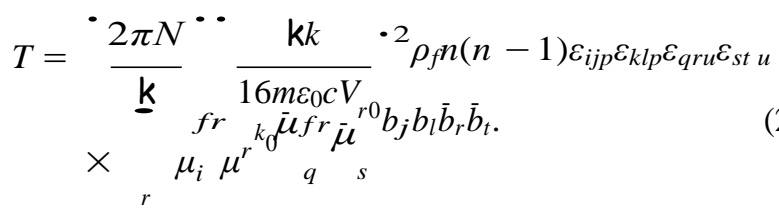

When the product of Levi-Civita tensors is evaluated using the tensor identity $\varepsilon_{i j p} \varepsilon_{k l p} \varepsilon_{q r u} \varepsilon_{s t} u=\delta_{i k} \delta_{j l} \delta_{q s} \delta_{r t}+$ $\delta_{i l} \delta_{j k} \delta_{q t} \delta_{r s}-\delta_{i k} \delta_{j l} \delta_{q t} \delta_{r s}-\delta_{i l} \delta_{j k} \delta_{q s} \delta_{r t}$, (23) becomes

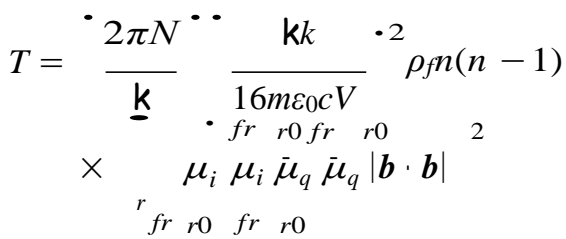

$$
\begin{aligned}
& -\mu_{i} \mu_{i} \bar{\mu}_{t} \bar{\mu}_{s}(\boldsymbol{b} \cdot \boldsymbol{b}) \bar{b}_{t} \bar{b}_{s}
\end{aligned}
$$

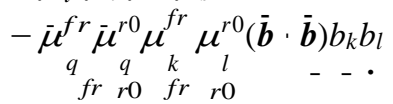

$$
\begin{aligned}
& +\mu_{k} \mu_{l} \quad \bar{\mu}_{t} \bar{\mu}_{s} b_{k} b_{l} b_{t} b_{s} \text {. }
\end{aligned}
$$

With the aid of standard techniques [20] we can now perform a rotational average on the rate, which is required when the orientations of the absorbing molecules are random, as in a gas or liquid. It is instructive to show the results of the averaging technique for each of the different rank tensors in 
Eq. (24). These are given as follows:

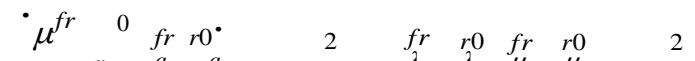

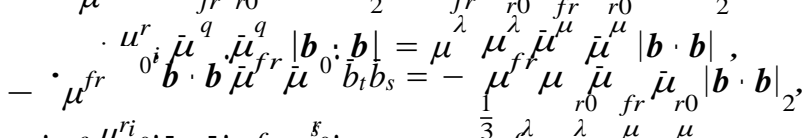

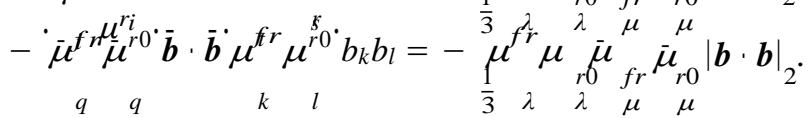

The final term within the bracket of Eq. (24) corresponds to a rank four tensor and, therefore, is more complicated to use in a rotational average procedure, as is shown below:

$$
\begin{aligned}
& { }_{k}^{f r} \mu_{l}^{r}{ }_{l}^{r}{ }_{t}{ }_{i} \bar{\mu}_{s}^{r 0} b_{k} b_{l} b_{t} b_{s}=b_{k} b_{l} b_{t} b_{s}-\left[\delta_{k l} \dot{\delta}_{t s}\left(4 \delta_{\lambda \mu} \delta_{v \pi}-\delta_{\lambda v} \delta_{\mu \pi}-\delta_{\lambda \pi} \delta_{\mu v}\right)+\delta_{k t} \delta_{l s}\left(-\delta_{\lambda \mu} \delta_{v \pi}+4 \delta_{\lambda v} \delta_{\mu \pi}-\delta_{\lambda \pi} \delta_{\mu v}\right)\right. \\
& \left.+\delta_{k s} \delta_{l t}\left(-\delta_{\lambda \mu} \delta_{v \pi}-\delta_{\lambda v} \delta_{\mu \pi}+4 \delta_{\lambda \pi} \delta_{\mu v}\right)\right] \mu_{\lambda}^{f r} \mu_{\mu}^{r 0} \bar{\mu}_{v}^{f r} \bar{\mu}_{\pi}^{r 0},
\end{aligned}
$$

Upon contracting the greek indices, (28) becomes

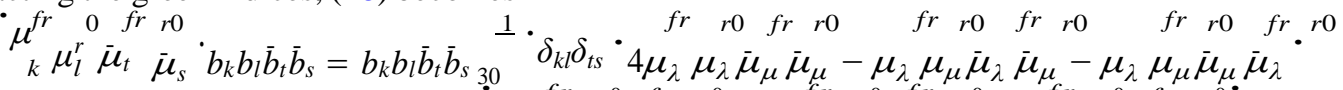

$$
\begin{aligned}
& +\delta_{k t} \delta l s-\mu^{f r} \mu^{r 0} \bar{\mu}^{f r} \bar{\mu}^{r 0}+4 \mu^{f r} \mu^{r 0} \bar{\mu}^{f r} \bar{\mu}^{r 0}-\mu^{f r} \mu^{r 0} \bar{\mu}^{f r} \bar{\mu}^{r 0}
\end{aligned}
$$

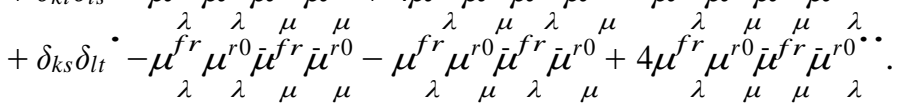

In all of the above, latin indices refer to the laboratory-fixed frame and the greek indices to the molecule-fixed frame.

Contracting the latin indices of Eq. (29) gives the final result as

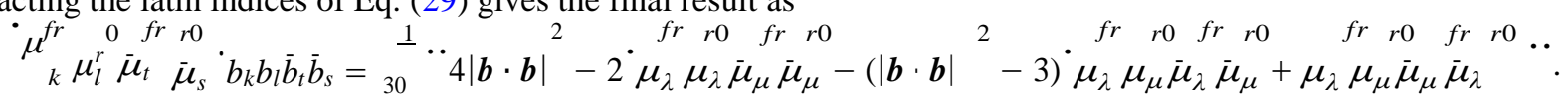

Inserting (25)-(27) and (30) into the rate equation (24) gives the final rotationally averaged result as

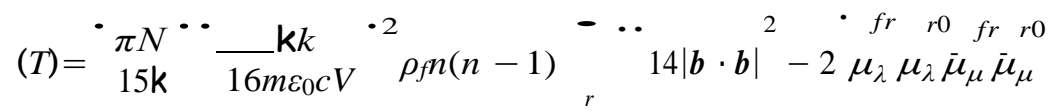

$$
\begin{aligned}
& \text { - }\left(|\boldsymbol{b} \cdot \boldsymbol{b}|_{2}-3\right)^{\cdot} \mu_{\lambda}^{f r} \mu_{\mu}^{r 0} \bar{\mu}_{\lambda}^{f r} \bar{\mu}_{\mu}^{r 0}+\underset{\lambda}{\mu f r} \mu_{\mu}^{r 0} \bar{\mu}_{\mu}{ }_{\mu} \bar{\mu}_{\lambda}^{r 0}{ }^{*} .
\end{aligned}
$$

The premultiplier can be rewritten more succinctly by taking into account the degree of second-order coherence $g^{(2)}$ [21], where $g^{(2)}=(n(n-1)) /(n)^{2}$, and then making use of the fact that the beam intensity may be given as the mean irradiance $\bar{I}=(n) c \mathrm{k} \omega / V$. The rate equation (31) finally becomes expressible as

$$
(T)=N \bar{I}^{2} g^{(2)} B^{\text {dia }},
$$

where the polarization-dependent molecular factor $B^{\text {dia }}$ is the diamagnetic two-photon analog of the Einstein $B$ coefficient, given explicitly as follows:

$$
B^{\text {dia }}=\frac{\pi \rho f}{3840 m^{2} c^{6} \mathrm{k} \varepsilon_{0}^{2}}{ }_{r}^{-} \cdot\left(14|\boldsymbol{b} \cdot \boldsymbol{b}|^{2}-2\right) \mu_{\lambda}^{f r} \mu_{\lambda}^{r 0} \bar{\mu}_{\mu}^{f r} \bar{\mu}_{\mu}^{r 0}-\left(|\boldsymbol{b} \cdot \boldsymbol{b}|^{2}-3\right) \mu_{\lambda}^{f r} \mu_{\mu}^{r 0} \bar{\mu}_{\lambda}^{f r} \bar{\mu}_{\mu}^{r 0}+\mu_{\lambda}^{f r} \mu_{\mu}^{r 0} \bar{\mu}_{\mu}^{f r} \bar{\mu}_{\lambda}^{r 0}{ }_{\lambda} .
$$

The absorption rate thus depends on the polarization of the incident radiation through the scalar product $(\boldsymbol{b} \cdot \boldsymbol{b})$, which is unity for linear polarization. Conversely this factor becomes zero for left or right circular polarization, for which we have $\boldsymbol{b}^{\mathrm{L} / \mathrm{R}}=(-i / 2)(\hat{\boldsymbol{i}} \pm i \hat{\boldsymbol{j}})$.

It is illuminating to compare the above result with the standard electric dipole-electric dipole result—as given by ${ }^{1}$

$$
(T)=N \bar{I}^{2} g^{(2)} B^{\mathrm{TPA}},
$$

where

$$
B^{\mathrm{TPA}}=\frac{\pi \rho_{f}}{120 \varepsilon_{0}^{2} \mathbf{k}} \cdot\left(\left.2 \boldsymbol{e} \boldsymbol{e}^{2}\right|^{f i}-1\right) \alpha_{\lambda \lambda}(\omega, \omega) \bar{\alpha}_{\mu \mu}^{f i}(\omega, \omega)-\left(|\boldsymbol{e} \cdot \boldsymbol{e}|^{2}-3\right) \alpha_{\lambda \mu}(\omega, \omega) \bar{\alpha}_{\lambda \mu}(\omega, \omega)
$$

\footnotetext{
${ }^{1} \mathrm{~A}$ minor difference from the result reported by Craig and Thirunamachandran is that our results are written explicitly in terms of the square of the input irradiance, reflecting our decision on the most appropriate form for the density of states factor.
}

In Eq. (35), $\alpha_{\lambda \lambda}^{f i}(\omega, \omega)$ is a scalar, the trace of the secondrank tensor $\alpha_{\lambda \mu}^{f i}(\omega, \omega)$; the latter is the usual (electric-dipole response) molecular two-photon absorption tensor. Although this tensor is not intrinsically index symmetric in its construction, it can be treated as such since only its indexsymmetric part can give a nonzero result when, in the detailed theory, it is duly coupled to the two equivalent input photon 


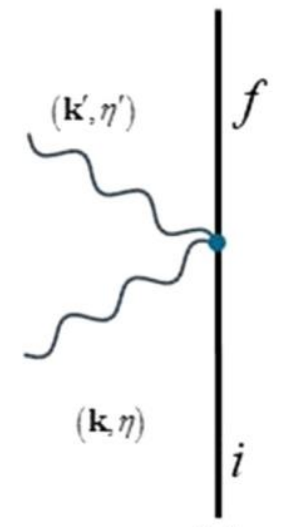

(a)

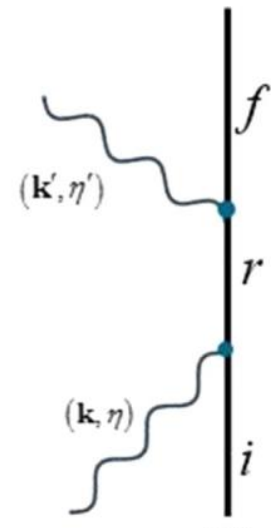

(b)
FIG. 2. (a) Feynman diagram for the diamagnetic contribution to Rayleigh scattering. (b) Conventional diagram for Rayleigh scattering.

polarization vectors. The standard selection rules for TPA are a consequence of this form. Comparing in more detail Eq. (33), it is evident that the selection rules with which the diamagnetization contribution is associated are determined by properties of the dyadic $\mu_{\lambda}^{f r} \mu_{\mu}^{r 0}$. Since it, too, acquires an effectively index-symmetric form due to its tensor contraction with measertif poldarization vectors, it yields precisely the same

\section{B. Application to Rayleigh scattering}

The two-photon scattering process involving one creation and one annihilation of a photon is studied here and illustrated by the Feynman diagrams of Fig. 2. Both Raman and Rayleigh scattering are types of two-photon scattering. As with TPA, nondiamagnetic leading contributions to the matrix element are of second order in the interaction, but diamagnetic contributions are first order in the interaction. The matrix element for the latter contribution to scattering is readily found to be

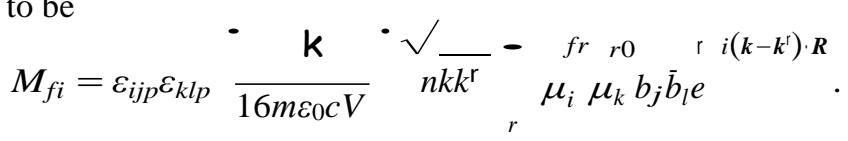

Using the Fermi rate rule together with [22] $\rho_{\text {emission }}=$ $k^{\mathrm{r} 2} d K^{\mathrm{r} V} V /(2 \pi)^{3} \mathrm{k} c$ (as it is now more appropriate to associate the degree of uncertainty with the precise axis of emission for the final state of the radiation field) allows us to express the scattering rate differential scattering cross section, using the infinitesimal scattering rate below:

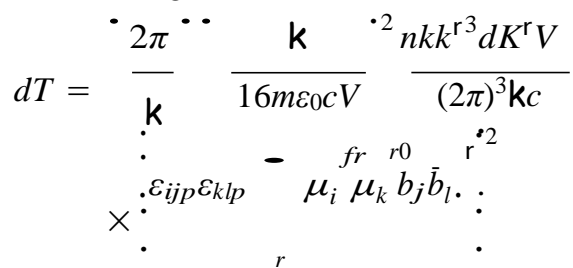

This rate may be converted into an infinitesimal cross section by dividing by the photon flux number $(n c / V)$ :

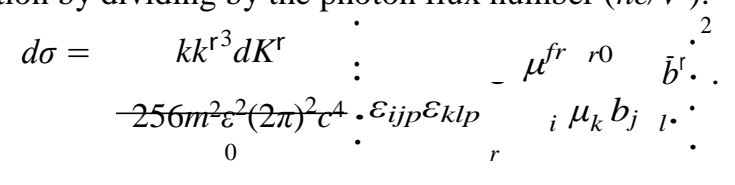

The differential cross section $d \sigma / d K^{\dagger}$ follows immediately which is the Kramers-Heisenberg dispersion formula. We can once again perform a rotational average on the result which yields

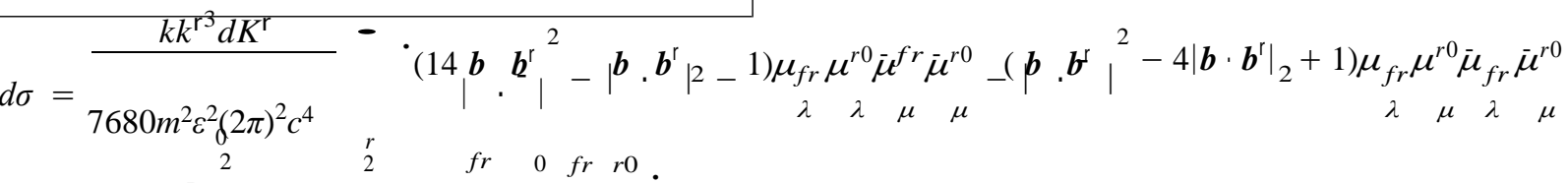

$$
\begin{aligned}
& -\left(\left|\boldsymbol{b} \cdot \overline{\boldsymbol{b}}^{\mathrm{r}}\right|^{2}+\left|\boldsymbol{b} \cdot \boldsymbol{b}^{\mathrm{r}}\right|^{2}-4\right) \mu_{\lambda} \mu_{\mu}^{r} \mu_{\mu} \bar{\mu}_{\mu} \bar{\mu}_{\lambda} \cdot
\end{aligned}
$$

Now using $I\left(\boldsymbol{k}^{\mathrm{r}}\right)=\frac{d T}{d K^{\mathrm{r}}} \mathrm{k} c k^{\mathrm{r}}$ and writing the result for an assembly of identical atoms non-forward scattering, we have

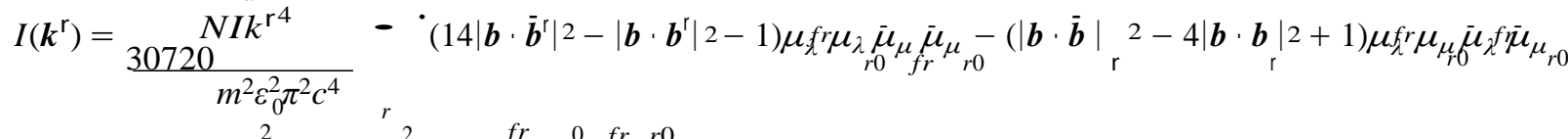

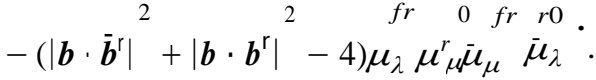

Finally, if we make the common assumption that the transition dipoles are real quantities, and express all of the Cartesian index contractions as scalar products, we can write, more simply,

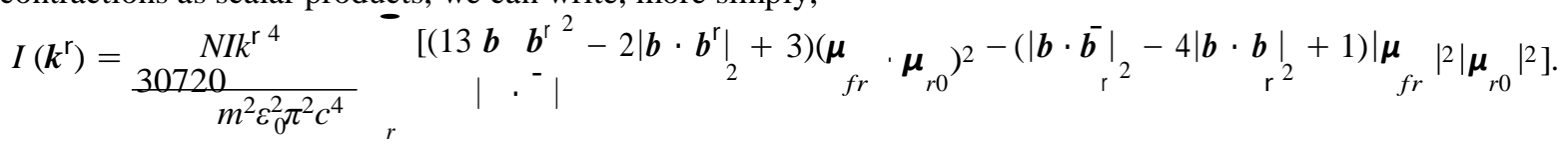

There is one especially striking difference between this result and the usual equation that emerges from electricdipole-only coupling, which is cast in terms of the molecular polarizability [16], the lack of a dispersion character, beyond the fourth power dependence on frequency (inverse fourth power of wavelength) that is a common hallmark of scattering intensities. The dispersion character that results from two dipole interactions is, in fact, a common feature associated 
with all conventional multipolar contributions. This means that in a study of Rayleigh scattering over any wavelength region where the kind of line shape arises that is associated with proximity to an electronic absorption band, or its wings, the diamagnetization contribution that we have identified is unique, and will be in principle analytically separable.

\section{DISCUSSION}

It has usually been argued that one of the most significant effects of diamagnetization coupling is a relatively small contribution to the dispersion interaction between molecules, in which it features alongside magnetic-dipole-magnetic-dipole, electric-quadrupole-electric-quadrupole, and the cross-term couplings. Elsewhere, inclusion of the diamagnetic coupling terms is justified on the basis that they are the same magnitude as other higher-order terms that are regularly studied [23-25] and in some cases are seen to be larger [26]. As indicated at the outset, this is commonly the primary basis for the inclusion of diamagnetic interactions; it leads to results that account properly for all processes within a given physical phenomenon. However, there are other features, of broader application, to which we can now draw further attention.

First, we have observed, with regard to its role in Rayleigh scattering, that the diamagnetization term delivers a contribution without the optical dispersion character with which that process is usually associated - as emerged in Eq. (41). Another interesting feature is the independence of the Rayleigh scattering intensity arising from diamagnetic coupling on wavelength; a similar behavior was obtained in the dispersion potential between two diamagnetic atoms [27]. In principle, a careful study of the wavelength dependence, over a region of significant dispersion, ought therefore to enable specific identification and quantification of the diamagnetization effect. This might be achieved, for example, by best-fitting the dispersion curve to a suitable line-shape function, running the residuals into a log-log plot against wavelength, and recognizing a -4 gradient. A similar effect could also, in principle, arise in two-photon absorption-if twin-beam excitation with two different optical frequencies were to be used (to allow a degree of freedom in the frequency input).

Secondly, there is one other aspect worthy of attention. In arriving at Eqs. (32) and (41), it may be observed that there is an underlying assumption-namely, that the usual free-space relationship between electric and magnetic field strengths applies. This is how the anticipated dependence on irradiance emerges. Indeed, by applying the relevant quantum operators it is readily shown that in their quadratic forms, each contributes exactly half of the $\left(n+\frac{1}{2}\right) \mathrm{k} \omega$ energy for a state with photon occupancy $n$, in a mode of frequency $\omega$. However, as a recent spate of publications [28-31] has shown, curious anomalies can arise with circularly polarized light, in the vicinity of a mirror upon which it has normal incidence. As a result of the superposition of forward and backward propagating light, the relative strengths of the electric and magnetic fields are then found to vary over distance, within the space of a wavelength. It thus transpires that for any observations of phenomena that can engage diamagnetization coupling with an input or reflected beam (such as the two-photon absorption studied above), the quadratic dependence on the magnetic field should manifest an exceptional position dependence, according to displacement from the mirror surface. A detailed analysis shows that diamagnetization contribution acquires a position-modified form, and the corresponding rate, as given by Eq. (32), becomes multiplied by a factor of $\cos ^{4} k d$, where $d$ is the distance from the mirror. In particular, it also emerges that the usually dominant electric-dipole form of interaction (involving the $\alpha$ tensor) is most strongly suppressed at the same location (due to quantum uncertainty the result is not exactly zero). In consequence, the diamagnetization response should dominate two-photon absorption at this position, alongside a weak magnetic-dipole-magnetic-dipole effect.

\section{ACKNOWLEDGMENT}

Financial support in the form of a UEA research studentship to K.A.F. is gratefully recorded.
[1] D. P. Craig, E. A. Power, and T. Thirunamachandran, Proc. R. Soc. A 322, 165 (1971).

[2] K. A. Forbes and D. L. Andrews, Phys. Rev. A 91, 053824 (2015).

[3] B. P. Krueger, G. D. Scholes, and G. R. Fleming, J. Phys. Chem. B 102, 5378 (1998).

[4] N. Tate, H. Sugiyama, M. Naruse, W. Nomura, T. Yatsui, T. Kawazoe, and M. Ohtsu, Opt. Express 17, 11113 (2009).

[5] P. Grahn, A. Shevchenko, and M. Kaivola, New J. Phys. 14, 093033 (2012).

[6] A. G. Curto, T.H. Taminiau, G. Volpe, M.P.Kreuzer, R. Quidant, and N. F. van Hulst, Nat. Commun. 4, 1750 (2013).

[7] D. J. Cho, F. Wang, X. Zhang, and Y.R. Shen, Phys. Rev.B 78, 121101 (2008).

[8] J. Petschulat, C. Rockstuhl, C. Menzel, A. Chipouline, A. Tünnermann, F. Lederer, and T. Pertsch, in Plasmonics and
Plasmonic Metamaterials, edited by G. Shvets and I. Tsukerman (World Scientific, Singapore, 2012), p. 67.

[9] A. Salam, J. Chem. Phys. 122, 044113 (2005).

[10] A. Salam, Phys. Rev.A 73, 013406 (2006).

[11] N. Moiseyev, M. Šindelka, and L. S. Cederbaum, Phys. Lett. A 362, 215 (2007).

[12] D. L. Andrews and J. M. Leeder, J. Chem. Phys. 130, 184504 (2009).

[13] S. Karaveli and R. Zia, Phys. Rev. Lett. 106, 193004 (2011).

[14] D. L. Andrews, Phys. Rev. A 81, 033825 (2010).

[15] M. Z. Alam, I. De Leon, and R. W. Boyd, Science 352, 795 (2016).

[16] D. P. Craig and T. Thirunamachandran, Molecular Quantum Electrodynamics: An Introduction to Radiation-Molecule Interactions (Dover Publications, Mineola, NY, 1998). 
[17] A. Salam, Molecular Quantum Electrodynamics. Long-Range Intermolecular Interactions (Wiley, Hoboken, NJ, 2010).

[18] M. Göppert-Mayer, Ann. Phys. (Berlin, Ger.) 401, 273 (1931).

[19] M. Pawlicki, H. A. Collins, R. G. Denning, and H. L. Anderson, Angew. Chem., Int. Ed. 48, 3244 (2009).

[20] D. L. Andrews and T. Thirunamachandran, J. Chem. Phys. 67, 5026 (1977).

[21] R. Loudon, The Quantum Theory of Light (Oxford University Press, Oxford, 2000).

[22] E. A.Power, Introductory Quantum Electrodynamics (American Elsevier Publishing Company, New York, 1965).

[23] U. I. Safronova, C. Namba, I. Murakami, W. R. Johnson, and M. S. Safronova, Phys. Rev.A 64, 012507 (2001).
[24] D. Tong, S. M. Farooqi, E. G. M. van Kempen, Z. Pavlovic, J. Stanojevic, R. Côté, E. E. Eyler, and P. L. Gould, Phys. Rev. A 79, 052509 (2009).

[25] J. Deiglmayr, H. Saßmannshausen, P. Pillet, and F. Merkt, Phys. Rev. Lett. 113, 193001 (2014).

[26] M. Marinescu and L. You, Phys. Rev. A 59, 1936 (1999).

[27] A. Salam, J. Phys. B: At., Mol. Opt. Phys. 33, 2181 (2000).

[28] Y. Tang and A. E. Cohen, Phys. Rev. Lett. 104, 163901 (2010).

[29] Y. Q. Tang and A. E. Cohen, Science 332, 333 (2011).

[30] E. Hendry, R. V. Mikhaylovskiy, L. D. Barron, M. Kadodwala, and T. J. Davis, Nano Lett. 12, 3640 (2012).

[31] M. M. Coles and D. L. Andrews, Opt. Lett. 38, 869 (2013). 